\section{Dementia and Geriatric} Cognitive Disorders
Accepted: February 10, 2010

Published online: April 6, 2010

\title{
Yamaguchi Fox-Pigeon Imitation Test: A Rapid Test for Dementia
}

\author{
Haruyasu Yamaguchi ${ }^{a}$ Yohko Maki ${ }^{a}$ b Tetsuya Yamagami ${ }^{a}$ \\ ${ }^{a}$ Gunma University School of Health Sciences, ${ }^{b}$ Department of Neurology, Geriatrics Research Institute \\ and Hospital, Maebashi, Japan
}

\section{Key Words}

Dementia • Alzheimer disease $\cdot$ Mild cognitive impairment •

Psychological burden $\cdot$ Gesture imitation

\begin{abstract}
Background/Aims: We herein propose a hand-gesture imitation test, consisting of a simple one-handed sign of a 'fox' and a complex two-handed sign of a 'pigeon', as a rapid, game-like test for detecting dementia/Alzheimer disease (AD) with low psychological burden. The test measures the visuomotor function, which deteriorates in the early stages of AD. Methods: We examined 88 demented subjects, 19 with mild cognitive impairment $(\mathrm{MCl})$, and 53 normal controls aged 65 years or over. The subjects were classified according to the Clinical Dementia Rating (CDR). Results: The specificity of the test was $94 \%$, and the sensitivity was $58 \%$ in CDR 0.5 (MCl), 77\% in CDR 1 (mild dementia), 75\% in CDR 2 (moderate dementia), and $90 \%$ in CDR 3 (severe dementia). The test could be conducted within $1 \mathrm{~min}$ and no subjects refused to be tested. Conclusion: This brief hand-gesture imitation test can sensitively evaluate visuomotor deficits in dementia/AD, while some subjects are unaware of their failure or even that their cognitive function is being tested. We herein describe the precise protocol for worldwide use.
\end{abstract}

Copyright $\odot 2010$ S. Karger AG, Basel

\section{Introduction}

As aging is a major risk factor for dementia, the rapid aging of society leading to large increases in people with dementia will result in a heavy socioeconomic burden. Thus, the early detection and prevention of dementia are urgently required.

We propose a hand-gesture imitation test, as a rapid game-like test, evaluating the visuomotor function for dementia/Alzheimer disease (AD). Although memory deficits are a salient feature of $\mathrm{AD}$, other symptoms appear from the early stages and a representative symptom is visuomotor deficit [1]. Before the onset of clinical symptoms, the bilateral parietal lobes are affected with $\mathrm{AD}$ related pathology [2], and show hypoperfusion as a characteristic single-photon emission computed tomography finding [3]. Thus, gesture imitation, which requires parietal function, may be compromised even in the early stages of $\mathrm{AD}$, a major cause of dementia.

Among gestures, we adopt meaningless nonsymbolic gestures to eliminate semantic components, although some studies insisted that meaningful imitation was more sensitive than meaningless imitation to detect $\mathrm{AD}$ [4-6]. However, meaningful imitation recruits semantic processing [7], and the semantic aspects of imitation are preserved in patients with lesions restricted to the pari-

\section{KARGER \\ Fax +4161306 1234 E-Mail karger@karger.ch} www.karger.com
Haruyasu Yamaguchi

Gunma University School of Health Sciences

3-39-15 Showa-machi

Maebashi 371-8514 (Japan)

Tel./Fax +81 27220 8946, E-Mail yamaguti@ health.gunma-u.ac.jp 
Table 1. Demographic data, error rate, and error patterns

\begin{tabular}{|c|c|c|c|c|c|c|c|c|c|c|}
\hline \multirow[t]{2}{*}{ Classification } & \multirow[t]{2}{*}{ CDR } & \multirow[t]{2}{*}{ Number } & \multirow{2}{*}{$\begin{array}{l}\text { Age } \\
\text { years }\end{array}$} & \multirow[t]{2}{*}{ HDS-R } & \multirow{2}{*}{$\begin{array}{l}\text { Error of } \\
\text { 'fox' } \\
n^{1}\end{array}$} & \multirow{2}{*}{$\begin{array}{l}\text { Error of } \\
\text { 'pigeon' } \\
\mathrm{n}^{1}\end{array}$} & \multicolumn{4}{|c|}{ Error patterns of 'pigeon' } \\
\hline & & & & & & & $\begin{array}{l}\text { palm-palm } \\
\mathrm{n}^{2}\end{array}$ & $\begin{array}{l}\text { palm- } \\
\text { dorsum, } \mathrm{n}^{2}\end{array}$ & $\begin{array}{l}\text { dorsum- } \\
\text { dorsum, } \mathrm{n}^{2}\end{array}$ & $\begin{array}{l}\text { interme- } \\
\text { diate, } \mathrm{n}^{2}\end{array}$ \\
\hline $\mathrm{NC}$ & 0 & 53 & $78.9 \pm 5.3$ & - & $0(0.0)$ & $3(5.7)$ & $3(100)$ & $0(0.0)$ & $0(0.0)$ & $0(0.0)$ \\
\hline MCI & 0.5 & 19 & $80.7 \pm 7.4$ & $26.5 \pm 1.3$ & $0(0.0)$ & $11(57.9)$ & $5(45.5)$ & $1(9.1)$ & $5(45.5)$ & $0(0.0)$ \\
\hline Mild dementia & 1 & 39 & $81.4 \pm 6.5$ & $20.2 \pm 4.1$ & $1(2.6)$ & $30(76.9)$ & $10(33.3)$ & $6(20.0)$ & $10(33.3)$ & $4(13.3)$ \\
\hline Moderate dementia & 2 & 20 & $82.1 \pm 5.4$ & $12.1 \pm 3.8$ & $0(0.0)$ & $15(75.0)$ & $11(73.3)$ & $3(20.0)$ & $1(6.7)$ & $0(0.0)$ \\
\hline Severe dementia & 3 & 29 & $81.6 \pm 4.0$ & $7.0 \pm 5.0$ & $9(31.0)$ & $26(89.7)$ & $0(0.0)$ & $11(42.3)$ & $12(46.2)$ & $3(11.5)$ \\
\hline Dementia total & $1-3$ & 88 & $81.6 \pm 5.5$ & $14.0 \pm 7.3$ & $10(11.4)$ & $71(80.7)$ & $21(23.9)$ & $20(22.7)$ & $23(26.1)$ & $7(8.0)$ \\
\hline
\end{tabular}

Figures in parentheses represent percentage. More than half of MCI (CDR 0.5) and 4/5 of demented subjects failed to imitate 'pigeon'. The most frequent error pattern of 'pigeon' was the palm-palm pattern, especially in CDR 0.5-2. HDS-R = Hasegawa's Dementia Scale-Revised (top score is 30 , as in the Mini-Mental State Examination).

${ }^{1}$ Errors as a percentage of the total number of attempts.

${ }^{2}$ Each error pattern as a percentage of the total number of errors.

etal lobe [8]. Meaningless gesture imitation examines representation of the body state [9], which is affected by parietal lobe deficits $[10,11]$. Thus, we tested meaningless imitation and adjusted the difficulty level according to a previous study showing that complex meaningless gesture imitation can detect $\mathrm{AD}$ in the early stages, whereas simple ones cannot [12].

Here, we developed an easy and rapid test, the Yamaguchi Fox-Pigeon Imitation Test (YFPIT), which detects dementia/AD within $1 \mathrm{~min}$. This game-like test reduces the psychological burden associated with ordinary cognitive tests, which often hurts the pride and self-confidence of aged people.

\section{Methods}

We tested 160 aged people: 97 in out-patient clinics and 63 in residences for seniors; 41 males and 119 females. The inclusion criterion was being aged 65 years or over. Exclusion criteria were psychiatric diseases, delirium, verbal incomprehension including aphasia, inability to walk, and motor deficits such as paralysis. The Ethics Board of Gunma University School of Health Sciences approved all procedures (No. 21-26), and signed informed consent was obtained from participants or their proxies. Subjects were diagnosed based on criteria for dementia diseases such as National Institute of Neurological and Communicative Disorders and Stroke and the Alzheimer's Disease and Related Disorders Association (NINCDS-ADRDA). Normal controls (NC) were judged by interviews and questionnaires on frequent symptoms of dementia and instrumental activities of daily living, taken from their family members/carers. Patients and some NC underwent MRI and a set of cognitive tests: e.g. Hasegawa's Dementia ScaleRevised, which is similar and well-correlated with the Mini-Mental State Examination and common in Japan, the cube copying test, clock drawing test, Stroop test, trail-making test, and memory tests. The demented subjects consisted of 64 with AD, 13 with dementia with Lewy bodies, and 11 with other dementia types. In the present study, subjects were classified according to the Clinical Dementia Rating (CDR; table 1). There were no significant differences in age nor gender among the groups (age: $p=0.11$, 1-way ANOVA; gender: $\mathrm{p}=0.19, \chi^{2}$ test).

The YFPIT consists of a hand-gesture imitation of a 'fox' (fig. 1a) contiguous with a 'pigeon' (fig. 1b). The protocol is as follows:

(1) The examiner sits face-to-face with a subject.

(2) The examiner gives a simple instruction: 'Watch my hand gesture carefully and imitate it.' The instruction can be repeated if necessary.

(3) Then, the examiner makes the 'fox' sign using his/her left hand: fingers III and IV touching the thumb on flexion of the metacarpophalangeal joints with fingers II and V held up (fig. 1a).

(4) The examiner maintains the gesture for $10 \mathrm{~s}$. The subject imitates the gesture concurrently with the examiner. Say nothing during the $10 \mathrm{~s}$ of the test. Be careful not to say the words 'fox', or the instruction.

(5) The examiner judges whether or not the subject produces the same sign within $10 \mathrm{~s}$ of demonstration; the subject may use either hand.

(6) For 'pigeon', the examiner gives the same instruction, and then makes a 'pigeon' sign using both hands: crossing the hands, palms facing the body, with fingers II-IV extended upward and the two thumbs crossing each other (fig. 1b).

(7) The examiner maintains the gesture without saying anything, especially the word 'pigeon' nor instructions, during the $10 \mathrm{~s}$ of the test.

(8) The examiner judges whether or not the subject concurrently makes the same sign within 10 seconds of demonstration.

Points for judgment are as follows:

(a) The direction of the arm and fingers II-V should be upward: hand positions in horizontal or downward directions are judged as failures (fig. 1f). 

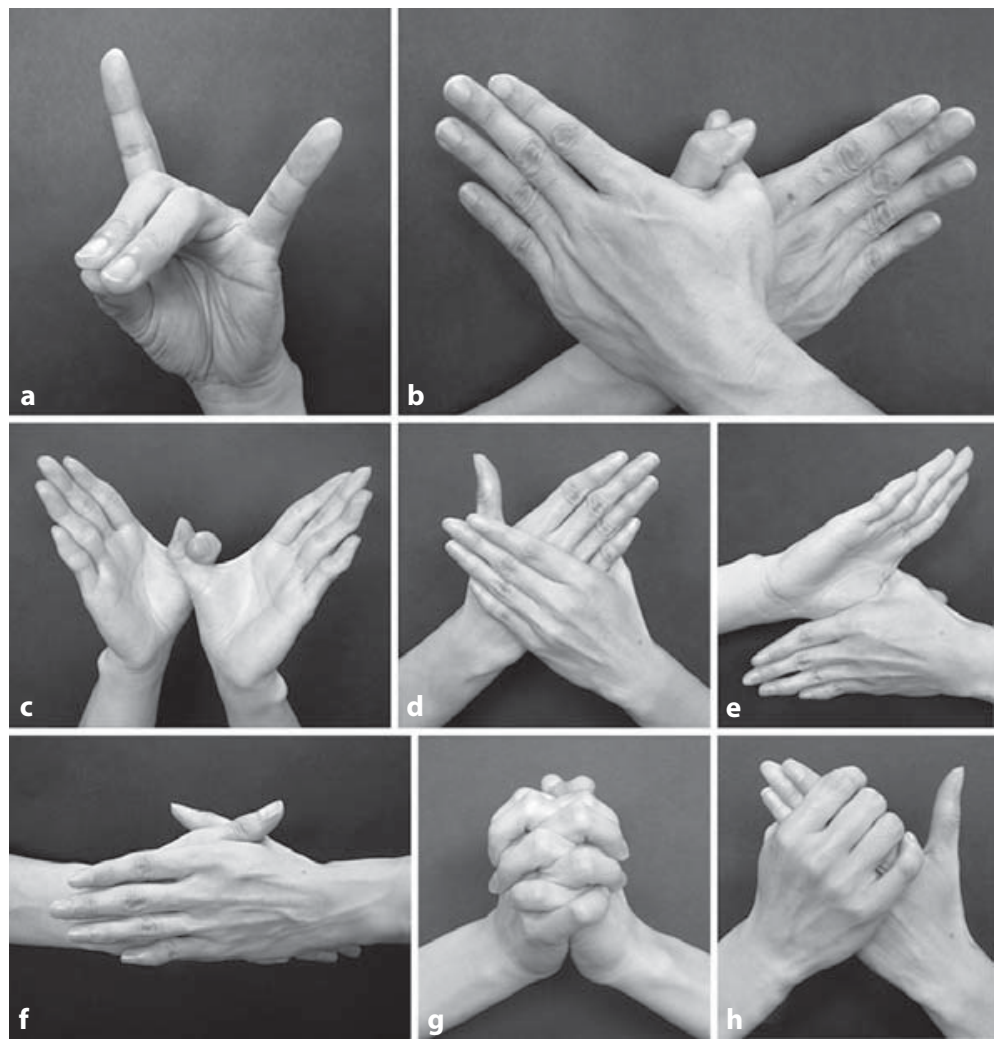

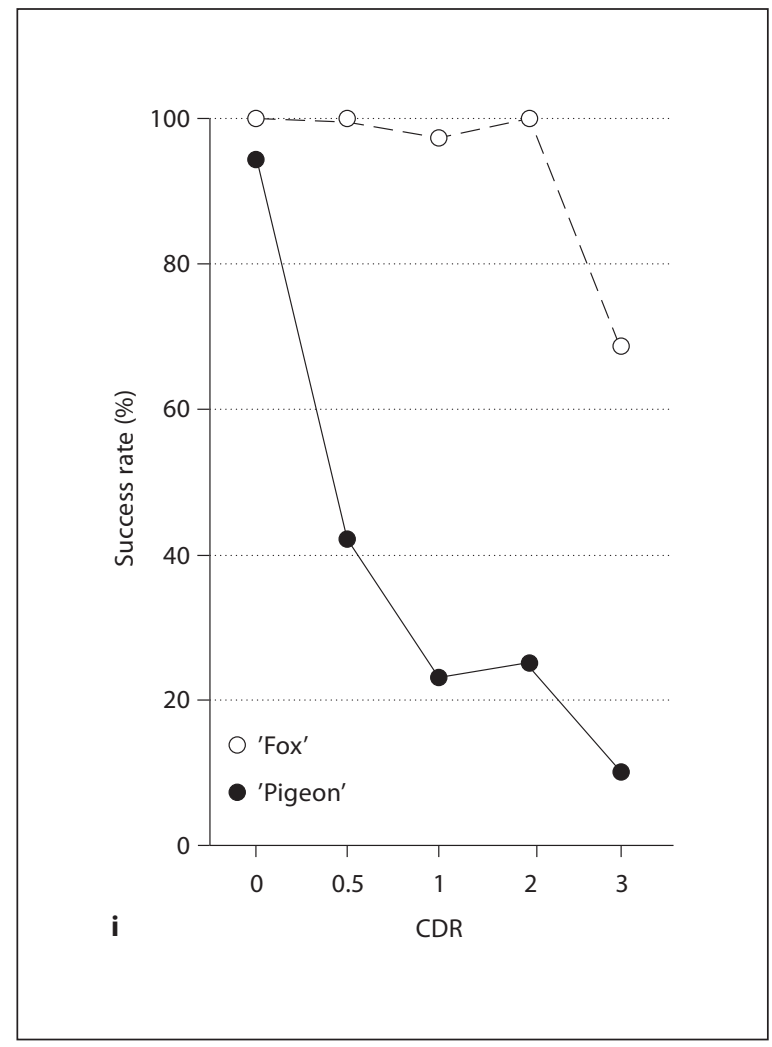

Fig. 1. Hand-gesture demonstration, error responses, and the success rate of the YFPIT according to the CDR. Examiner's demonstration of 'fox' (a) and 'pigeon' (b). Subjects' typical error patterns of palm-palm (c), dorsumdorsum (d), and palm-dorsum (e). f Error pattern with downward direction of arms. g, h Error patterns common in CDR 3. i The success rate of 'fox' started to decrease at CDR 3, while that of 'pigeon' decreased at CDR 0.5.

(b) Both cases are acceptable: right hand in an outward position or vice versa.

(c) Both palms should be facing the body.

(d) Thumbs should be crossing each other.

\section{Results}

Most CDR 0-2 subjects succeeded in imitating the 'fox', whereas $31.0 \%$ of CDR 3 subjects failed. No subjects who failed to imitate the 'fox' succeeded with the 'pigeon' (fig. $1 \mathrm{i}$ and table 1).

On the other hand, the success rate of the 'pigeon' was $94.3 \%$ in NC (i.e. specificity), whereas more than half of the subjects with MCI (CDR 0.5) and 4/5 of demented subjects failed to imitate 'pigeon' (table 1). The error rate was not significantly influenced by age ( $\mathrm{p}=0.17$, twosample $t$ test) nor gender $\left(\mathrm{p}=0.10, \chi^{2}\right)$. The specificity of the test was $94.3 \%$. In comparison between NC and MCI, the sensitivity was $57.9 \%$, the positive predic- tion value (PV+; positive diagnosis/test positive) was $86.2 \%$, and negative prediction value (PV-; negative diagnosis/test negative) was $78.6 \%$. In comparison between NC and mild dementia, the sensitivity was 76.9\%, PV+ was $84.7 \%$, and PV- was $90.9 \%$. When subjects were limited to the 64 with $\mathrm{AD}$ and compared to $\mathrm{NC} / \mathrm{MCI}$, the results were similar to those obtained from all demented subjects (online suppl. table 1, www. karger.com/doi/10.1159/000289819). The number of subjects was too small to analyze the differences among the causes of dementia.

As qualitative analysis, we categorized 4 error patterns of 'pigeon' based on the direction of the hands: (1) palmpalm pattern, both palms outward (fig. 1c), (2) dorsumdorsum pattern, both dorsa outward (fig. 1d), (3) palmdorsum pattern, one palm and one dorsum outward (fig. 1e), and (4) other patterns. The characteristic error pattern was palm-palm, and subjects showing this error seemed not to notice it, because, from the subjects' per- 
spective, they saw their dorsa as well as the dorsa of the examiner (table 1).

Regardless of the hand direction, severely demented subjects showed a tendency to fold their fingers $(n=12$, 41.4\% in CDR 3), e.g. bring their hands together (fig. 1g), or grip one hand with the other (fig. 1h). Inability to perform 'fox' is also observed mostly among CDR 3 subjects, and, therefore, the rate of subjects showing a folding finger pattern and/or inability to perform 'fox' was $0 \%$ in CDR $0.5,5.1 \%$ in CDR $1(\mathrm{n}=2)$, and $5.0 \%$ in CDR $2(\mathrm{n}=$ $1)$, whereas it was $58.6 \%$ in CDR $3(n=17)$.

No subjects refused to undertake the test and all finished the test within $1 \mathrm{~min}$.

\section{Discussion}

Our results suggest that the YFPIT is useful to detect dementia/AD. The merits of the YFPIT are:

(1) Easy and simple, requiring $1 \mathrm{~min}$.

(2)Enjoyable, like a game.

(3) Nearly half of the subjects with mild to moderate dementia showed a palm-palm pattern, and did not notice their mistakes, preserving self-confidence.

(4) Low error rate of 'pigeon' in NC (5.7\%).

(5) High sensitivity for detecting dementia (80.7\%).

Adding to the merits for detecting mild dementia, the YFPIT revealed the characteristics of severe dementia; more than half of CDR 3 subjects failed to imitate 'fox' and/or showed a folding finger pattern (i.e. fig. $1 \mathrm{~g}$, h) in 'pigeon'.

Why did the YFPIT sensitively detect dementia? The reason is that we adopted a hand gesture, 'pigeon', containing 2 components as follows. One was the component of perspective taking, which is the cognitive process when perceiving a visual scene from one's own perspective (first-person perspective, 1PP), differing from taking a view of the same scene from another person's viewpoint (third-person perspective, 3PP) [13]; 3PP recruits the bilateral parietal area more intensely than $1 \mathrm{PP}$ [14]. The most frequent error pattern was the palm-palm pattern, which is related to deficits of perspective taking. In the palm-palm pattern, the subjects see the dorsum of both the examiner and themselves. Another component is body midline crossing, where the hands invade the contralateral space. Making adequate gestures crossing the midline is more complex than making those limited to ipsilateral sides.

As above, with the YFPIT, we successfully detected dementia/AD in the early stages. However, a detection (er- ror) rate of $58 \%$ in MCI (CDR 0.5) is insufficient. We do not propose that dementia should be screened with the YFPIT independently. The YFPIT can be one of the components of a test battery for dementia/AD. Questionnaires on instrumental activities of daily living from carers are also important for detecting dementia in its early stages [15]. Combination with other tests is necessary for an accurate diagnosis.

The main issue of $\mathrm{MCI}$ is the prognosis of conversion to AD. Imaging studies have reported that hypometabolism and/or hypoperfusion in parietal association areas have a high predictive value of conversion to $\mathrm{AD}$ [3], and hand-gesture imitation recruits the bilateral parietal association area. Thus, we assume that the hand-gesture imitation test is useful to predict the conversion from $\mathrm{MCI}$ to AD. To evaluate the prognostic role of the YFPIT in MCI, follow-up observation is needed.

Corticobasal degeneration is a progressive movement disorder with cortical and basal ganglionic dysfunction [16], and the hand-gesture imitation test is applied to evaluate the movement disorder [17]. Therefore, failure in the YFPIT can be seen in limb apraxia of corticobasal degeneration as well as ideomotor apraxia of stroke.

Our priority was to devise a simple test protocol - one easy gesture followed by a complex gesture, the difficulty of which is appropriate to distinguish dementia/AD from NC. As a result, more than half of CDR 0.5 subjects failed 'pigeon'. Gesture order is also important. We tested the simple 'fox' before the complex 'pigeon' gesture to rule out deficits caused by the incomprehension of verbal commands or visual deficits. To perform the test as a nonsymbolic imitation, we did not use the words 'fox' and 'pigeon'. Thus, the test evaluated the visuomotor but not semantic function. Previous studies revealed deficits of imitation in $\mathrm{AD}$ in the context of apraxia [4-7]. This hand-gesture imitation test, the YFPIT, is an effective 1-min test of dementia/AD, showing good sensitivity/ specificity, even though it is quite easy, rapid, and low in psychological burden.

\section{Acknowledgements}

This work was performed with no funding. However, Dr. Yamaguchi is supported by a grant-in-aid from the Ministry of Education, Science, Culture and Sports, Japan. Dr. Maki had salary support from the Good Practice Program of the Ministry of Education, Science, Culture and Sports, Japan. We thank Rumi Shinohara, and Yuko Tsunoda, research assistants at Gunma University School of Health Sciences, for help with the initial data analysis. 


\section{References}

1 Tippett WJ, Sergio LE: Visuomotor integration is impaired in early stage Alzheimer's disease. Brain Res 2006;1102:92-102.

$\checkmark 2$ Braak H, Braak E: Frequency of stages of Alzheimer-related lesions in different age categories. Neurobiol Aging 1997;18:351357.

3 Matsuda H: Role of neuroimaging in Alzheimer's disease, with emphasis on brain perfusion SPECT. J Nucl Med 2007;48:12891300.

4 Parakh R, Roy E, Koo E, Black S: Pantomime and imitation of limb gestures in relation to the severity of Alzheimer's disease. Brain Cogn 2004;55:272-274.

5 Blondel A, Desgranges B, de la Sayette V, Schaeffer S, Benali K, Lechevalier B, Viader F, Eustache F: Disorders in intentional gestural organization in Alzheimer's disease: combined or selective impairment of the conceptual and production systems? Eur J Neurol 2001;8:629-641.

-6 Chainay H, Louarn C, Humphreys GW: Ideational action impairments in Alzheimer's disease. Brain Cogn 2006;62:198-205.
Rumiati RI, Weiss PH, Tessari A, Assmus A, Zilles K, Herzog H, Fink GR: Common and differential neural mechanisms supporting imitation of meaningful and meaningless actions. J Cogn Neurosci 2005; 17:1420-1431.

8 Freund HJ: The parietal lobe as a sensorimotor interface: a perspective from clinical and neuroimaging data. Neuroimage 2001; 14:S142-S146.

9 Chaminade T, Meltzoff AN, Decety J: An fMRI study of imitation: action representation and body schema. Neuropsychologia 2005;43:115-127.

10 Wolpert DM, Goodbody SJ, Husain M: Maintaining internal representations: the role of the human superior parietal lobe. Nat Neurosci 1998;1:529-533.

11 Goldenberg G: Apraxia and the parieta lobes. Neuropsychologia 2009;47:14491459.

12 Derouesné C, Lagha-Pierucci S, Thibault S, Baudouin-Madec V, Lacomblez L: Apraxic disturbances in patients with mild to moderate Alzheimer's disease. Neuropsychologia 2000;38:1760-1769.

13 Vogeley K, Fink GR: Neural correlates of the first-person-perspective. Trends Cogn Sci 2003;7:38-42.
14 David N, Bewernick BH, Cohen MX, Newen A, Lux S, Fink GR, Shah NJ, Vogeley K: Neural representations of self versus other: visual-spatial perspective taking and agency in a virtual ball-tossing game. J Cogn Neurosci 2006;18:898-910.

15 Mariani E, Monastero R, Ercolani S, Rinaldi $\mathrm{P}$, Mangialasche F, Costanzi E, Vitale DF, Senin U, Mecocci P, ReGAl Study Group: Influence of comorbidity and cognitive status on instrumental activities of daily living in amnestic mild cognitive impairment: results from the ReGAl project. Int J Geriatr Psychiatry 2008;23:523-530.

16 Peigneux P, Salmon E, Garraux G, Laureys S, Willems S, Dujardin K, Degueldre C, Lemaire C, Luxen A, Moonen G, Franck G, Destee A, Van der Linden M: Neural and cognitive bases of upper limb apraxia in corticobasal degeneration. Neurology 2001;57: 1259-1268.

17 Salter JE, Roy EA, Black SE, Joshi A, Almeida Q: Gestural imitation and limb apraxia in corticobasal degeneration. Brain Cogn 2004; 55:400-402. 